\title{
THE PERMUTATION PRINCIPLE \\ IN QUANTIFICATIONAL LOGIC ${ }^{1}$
}

The story goes back to 1940, with the publication of Quine's Mathematical Logic [5]. He there presents a system of quantificational logic in which only sentences or closed formulas are theorems. In that system, the sole rule of inference is Modus Ponens and the axioms are the universal closures of the following formulas:

$$
\begin{aligned}
& \phi, \text { for } \phi \text { tautologous; } \\
& \forall \alpha(\phi \supset \psi) \supset(\forall \alpha \phi \supset \forall \alpha \psi) ; \\
& \phi \supset \forall \alpha \phi, \text { for } \alpha \text { not free in } \phi ; \\
& \forall \alpha \phi(\alpha) \supset \phi(\beta), \text { with usual restrictions; } \\
& \forall \alpha \forall \beta \phi \supset \forall \beta \forall \alpha \phi .
\end{aligned}
$$

Suppose the variables, in alphabetic order, are $x_{1}, x_{2}, \ldots$. Then Quine takes the universal closure of a formula $\phi$ to be the result of prefixing the quantifiers $\forall \alpha$, for $\alpha$ free in $\phi$, to that formula in their reverse of anti-alphabetic order.

The presence of the Permutation Principle (5) somewhat mars the elegance of the system; but Berry [1] was able to show how to do without it. Alter the definition of closure: prefix the universal quantifiers in their alphabetic, not their anti-alphabetic, order. The change is small and seemingly insignificant. But taking as axioms the alphabetic closures of (1)-(4) suffices to prove all theorems of the original system, including Permutation.

This change was incorporated into the second edition of Mathematical Logic [6]. But the question remained as to whether it was essential. Could axiom-scheme (5) also be dropped from the original system without any loss of theorems?

The answer is no. Let us define a certain transform $(\phi)^{t}$ of a formula $\phi$. Call the universal formula $\forall \alpha \psi$ non-vacuous if $\alpha$ occurs free in $\psi$. Given a subformula occurrence $\psi$ of $\phi$, say that $\psi$ is bound in $\phi$ if a free variable $\alpha$ of $\psi$ is bound by a quantifier $\forall \alpha$ outside of $\psi$ but in $\phi$. Now call the subformula occurrence $\psi$ of $\phi$ replaceable if (i) it is a non-vacuous formula of the form $\forall x_{1} \times$ (note the specific variable), (ii) it is bound in $\phi$, and (iii) it is 
not a proper subfermula of any other formula satisfying (i) and (ii). Then we may define the transform $(\phi)^{t}$ of $\phi$ to be the result of replacing all replaceable subformula occurrences in $\phi$ with $\perp$.

Let $Q$ be Quine's original system and let $Q^{-}$be the result of dropping axiom-scheme (5) from $Q$. Then it may be shown by an induction on proofs that:

LEMMA 1 . For any theorem $\phi$ of $Q^{-}$, the transform $(\phi)^{t}$ is classically valid.

Consider now the formula $\phi=\forall x_{1} \forall x_{2} F x_{1} x_{2} \supset \forall x_{2} \forall x_{1} F x_{1} x_{2}$. Then its transform $(\phi)^{t}$ is $\forall x_{1} \forall x_{2} F x_{1} x_{2} \supset \forall x_{2} \perp$, which is not classically valid. It therefore follows from Lemma 1 that

THEOREM 2. The formula $\forall x_{1} \forall x_{2} F x_{1} x_{2} \supset \forall x_{2} \forall x_{1} F x_{1} x_{2}$ is not a theorem of $Q^{-}$, and hence the axiom-scheme (5) is not redundant in the original system $Q$.

It should be clear that the non-derivability of many other instances of the scheme could be established in the same way.

Although the above formula $\phi$ is not derivable within $Q^{-}$, the formula $\forall x_{2} \forall x_{3} F x_{2} x_{3} \supset \forall x_{3} \forall x_{2} F x_{2} x_{3}$ is. Indeed, given any formula $\phi$, let $\phi^{n}$, for $n \geqslant 0$, be the result of replacing each variable $x_{i}$ in $\phi$ with $x_{n+i}$. Then it may be shown by an easy induction that if $\phi$ is a theorem of $Q$ then $\phi^{n}$ is a theorem of $Q^{-}$for some nonnegative integer $n$; though whether $\phi^{n}$ is derivable for a particular $n$ would seem to depend upon the availability of variables alphabetically earlier to those appearing in $\phi$ in a way that is hard precisely to determine.

This observation has a curious consequence in regard to the completeness of $Q^{-}$. The original system $Q$ is complete; each valid sentence is a theorem. The defective system $Q^{-}$is not complete, since instances of Permutation are not derivable. However, $Q^{-}$is complete up to relettering of variables. Given any valid sentence $\phi, \phi$ is derivable within $Q$ and hence an alphabetic variant $\phi^{n}$ of $\phi$ is derivable within $Q^{-}$.

The story now goes to 1963 , with the publication of papers by Kripke [2] and Lambert [3]. Kripke was concerned to block the derivation of the Barcan formula or its converse within a quantified version of the modal logic S5. He was able to do this by requiring, as in Quine [6], that only closed formulas be theorems. However, because he wished to dispense with 
the rule of necessitation and because he also wished to allow for the empty domain, he did not quite take Quine's revised system as the quantificational basis for his modal logic. Instead, he made two changes. First, he loosened up the definition of universal closure. He did not require of a universal closure $\forall \alpha_{1} \forall \alpha_{2} \ldots \forall \alpha_{n} \varphi$ of $\varphi$ that the quantifiers $\forall \alpha_{1}, \forall \alpha_{2}, \ldots, \forall \alpha_{n}$ be prefixed in any particular order or that their variables $\alpha_{1}, \alpha_{2}, \ldots, \alpha_{n}$ be free; he only required that the resulting formula $\forall \alpha_{1} \forall \alpha_{2} \ldots \forall \alpha_{n} \varphi$ be closed. Second, he took as his axioms the closures, in this sense, of (1), (2) and (3), and the following variant of (4):

$$
\forall \beta(\forall \alpha \varphi(\alpha) \supset \varphi(\beta)) \text {, with usual restrictions. }
$$

Quite independently, Lambert developed a similar system. Like Kripke, he was concerned to allow for the empty domain; but he also wished to allow for theorems with free variables. He therefore proposed, in effect, that the formulas (1), (2), (3) and (4), themselves be axioms (not their closures) and that both Modus Ponens and Generalization be taken as the rules of inference. To these axioms and rules, he then considered the result of adding the standard classical axioms for identity.

As later became clear, Lambert's full system (with identity) is complete for its intended interpretation. But it was then generally assumed that this system without its identity axioms and the corresponding quantificational part of Kripke's system (which had not been formulated with identity in the first place) were also complete. Indeed, in their paper [4] of 1970, Leblanc and Meyer gave a metalogical investigation of the Lambert fragment in which it was presupposed that Permutation and related principles were derivable; and, in [2], Kripke claimed completeness for his full modal system, which would have entailed completeness for its quantificational fragment. But then, Lambert pointed out, in a letter to Meyer of around 1968-9, the difficulty of deriving Permutation within the identity-free part of his system; and independently, in his paper of 1970 ([7], p. 286, fn. 6), Trew pointed to the related difficulty of deriving Permutation within Kripke's system. The problem of deriving the principle became open and, at least within the world of free logicians, achieved some notoriety.

It now appears that Permutation is not derivable within these systems. To see this, we construct a somewhat devious semantics for quantificational logic. Assume, for simplicity, that dyadic $F$ is the sole predicate in our language. Let a sensitive model $M$ for that language be a quadruple 
$(A, R, f, g$ ), where $A$ (domain) is a non-empty set, $R$ (extension of $\mathrm{F}$ ) is a subset of $A \times A$, and both $f$ and $g$ are functions from $A$ into $\mathrm{P}(A)$, satisfying the conditions that $a \in f(a)$ and $a \in g(a)$ for all $a \in A$. The pair $(A, R)$ may be regarded as a classical model. What $f$ and $g$ add to this model are relationships of dependence in the assignment of values. If, in the formula $F \alpha \beta, a$ has been assigned to $\alpha$, then only $b$ from $\mathrm{f}(a)$ can be assigned to $\beta$ (for the formula $\mathrm{F} \alpha \beta$ to be satisfied by the resulting assignment); and likewise, if $b$ has been assigned to $\beta$, only $a$ form $\mathrm{g}(b)$ can be assigned to $\alpha$.

We make this idea precise as follows. Let $M=(A, R, f, g)$ be a sensitive model. Add all objects of $A$ to the language as names of themselves. Let the standard substitution $\phi^{a} / \alpha$ of a for $\alpha$ in $\phi$, with $a \in A$, be the result of replacing all free occurrences of $\alpha$ in $\phi$ with $a$. Let the deviant substitution $\phi^{a} \xi \alpha$ be obtained from the standard substitution $\varphi^{a} / \alpha$ in two complementary steps. First, put $\perp$ for all those occurrences of $F a b$ in $\varphi^{a} / \alpha$ that come from $\mathrm{F} \alpha b$ in $\varphi$ upon replacing the free occurrence of $\alpha$ in $\mathrm{F} \alpha b$ with $a$ and for which $b \in A$ but $a \notin \mathrm{g}(b)$; let the result be $\psi$. Now put $\perp$ for all those occurrences of $\mathrm{F} b a$ in $\psi$ that come from $\mathrm{F} b \alpha$ in $\varphi$ upon replacing the free occurrence of $\alpha$ in $\mathrm{F} b \alpha$ with $a$ and for which $b \in A$ but $a \notin \mathrm{f}(b)$.

Define deviant truth by the following clauses:

$$
\begin{aligned}
& M \vDash \mathrm{F} a b \text { iff }\langle a, b\rangle \in R ; \\
& M \vDash-\phi \text { iff not } M=\phi ; \\
& M \vDash(\phi \vee \psi) \text { iff } M \vDash \phi \text { or } M \vDash \psi ; \\
& M \vDash \forall \alpha \phi \text { iff } M \vDash \phi_{s^{3}}^{a} \alpha \text { for all } a \in A .
\end{aligned}
$$

Say that a formula $\phi$ is true in $M$ if the result of substituting any elements of $A$ for the free variables of $\phi$ is true in $M$.

It may now be shown by a tedious induction on proofs that:

LEMMA 3. All the theorems of Lambert's system, and hence of Kripke's system too, are true in any sensitive model.

It is instructive to see why the proof breaks down for the systems with identity. Take the identity axiom $\varphi=\forall \alpha \forall \gamma \forall \beta(\alpha=\beta \supset(F \alpha \gamma \supset F \beta \gamma))$, for $\alpha, \beta$ and $\gamma$ distinct variables. Then relative to the model $M$ above, $\varphi 1 / \alpha^{0} / \gamma^{1} \beta=(1=1 \supset(\mathrm{F} 10 \supset \perp))$ and hence is false.

Consider now the following sensitive model $M=(A, R, f, g)$ : 


$$
\begin{aligned}
& A=\{0,1\} ; \quad R=A \times A ; \quad f(0)=f(1)=A ; \\
& g(0)=\{0\} \text { and } g(1)=\{1\} .
\end{aligned}
$$

Take distinct variables $\alpha$ and $\beta$. Then $M \vDash \forall \alpha \forall \beta F \alpha \beta$, since $\mathrm{F} \alpha \beta_{\beta}^{a} \alpha^{b} \xi \beta=\mathrm{F} a b$ for all $a, b \in A$. On the other hand, $M \not \models \forall \beta \forall \alpha \mathrm{F} \alpha \beta$, since $\mathrm{F} \alpha \beta_{3}^{0} \beta \beta_{\xi}^{1} \alpha=1$.

From this and Lemma 3 follows the conclusion to our story:

THEOREM 4. The sentence $\forall \alpha \forall \beta F \alpha \beta \supset \forall \beta \forall \alpha F \alpha \beta$, for $\alpha$ distinct from $\beta$, is not derivable within the systems of Kripke and of Lambert.

University of Michigan

\section{NOTE}

2 I have been helped, in writing this paper, by the kind advice and encouragement of Karel Lambert and Hugues Leblanc.

\section{REFERENCES}

[1] Berry, G. D. W., 'On Quine's axioms of quantification', Journal of Symbolic Logic 6 (1941), 23-27.

[2] Kripke, S., 'Semantical considerations on modal logic', Acta Philosophica Fennica 16 (1963), 83-94.

[3] Lambert, K., 'Existential import revisited, Notre Dame Journal of Formal Logic 4 (1963), 288-292.

[4] Leblanc, H. and Meyer, R. K., 'On prefacing $(\forall X) A \supset A Y / X$ with $(\forall Y)-A$ free quantification theory without identity', Zeitschrift für Mathematische Logik und Grundlagen der Mathematik 16 (1970), 447-462.

[5] Quine, W. V., Mathematical Logic (1 st edn.), Harvard University Press, Boston, 1940.

[6] Quine, W. V., 2nd cdn. of above (1951).

[7] Trew, A., 'Nonstandard theories of quantification and identity', Journal of Symbolic Logic 35 (1970), 267-294. 